Research Article

\title{
Bearing Fault Diagnosis Using Synthetic Quantitative Index-Based Adaptive Underdamped Stochastic Resonance
}

\author{
Baochen Li $\mathbb{D}^{1},{ }^{1}$ Rui Tong $\left(\mathbb{D},{ }^{2}\right.$ Jianshe Kang $\mathbb{D}^{2},{ }^{2}$ and Kuo Chi $\mathbb{D D}^{3}$ \\ ${ }^{1}$ Office of Research and Development, Army Engineering University, Nanjing 210000, China \\ ${ }^{2}$ Shijiazhuang Branch, Army Engineering University, Shijiazhuang 050000, China \\ ${ }^{3}$ China Satellite Maritime Tracking and Control Department, Wuxi 214431, China \\ Correspondence should be addressed to Kuo Chi; chimingshu@foxmail.com
}

Received 16 September 2020; Revised 1 January 2021; Accepted 24 January 2021; Published 15 February 2021

Academic Editor: Francesco Riganti-Fulginei

Copyright ( $\odot 2021$ Baochen Li et al. This is an open access article distributed under the Creative Commons Attribution License, which permits unrestricted use, distribution, and reproduction in any medium, provided the original work is properly cited.

Stochastic resonance is like a nonlinear filter to detect the weak bearing fault-induced impulses that submerged in strong noises. Signal-to-noise ratio (SNR) is often used as the index to evaluate the SR output, but the fault characteristic frequency (FCF) must be known in order to calculate SNR. A novel bearing fault diagnosis method called synthetic quantitative index-based adaptive underdamped stochastic resonance (SQI-AUSR) is proposed. The synthetic quantitative index (SQI) is composed of power spectrum kurtosis, kurtosis, margin index, and correlation coefficient. The SQI is independent of FCF, which avoids the limitation that the calculation of SNR must know the FCF. Numeric simulations and two case studies of bearing faults are carried out. The results show that (1) the SQI is more effective than other proposed indexes such as correlation coefficient and weight power spectrum kurtosis and (2) the proposed SQI-AUSR is effective for bearing fault diagnosis and is better than SNR-AOSR.

\section{Introduction}

Bearing is one of the most commonly used parts in rotary machinery, but prone to failure because of its poor work environment such as high temperature, low lubrication, and strong cyclic stress, which can cause tremendous economic losses and even fatal accident. However, the impulses induced by bearing failure, especially the incipient bearing failure, are submerged in strong noise and difficult to be detected. To improve the weak fault-induced impulses, some methods are proposed. Bessous et al. [1] applied discrete wavelets transform to bearing defects' diagnosis. Chegini et al. [2] used empirical wavelet transform to reduce the noise of bearing vibration signal so as to realize bearing fault diagnosis. Gong et al. [3] proposed the tentative variational mode decomposition for the fault feature detection of rolling element bearing. Although much research has been carried out on bearing fault diagnosis, most methods focus on the noise removal rather than the faultinduced impulse enhancement.

Unlike the traditional filters, stochastic resonance (SR) is like the nonlinear filter that enhances the weak signal with the help of appropriate noise in the proper nonlinear system. However, according to adiabatic approximation theory, the traditional SR only can process the small-parameter signal that the frequency is less than $1 \mathrm{~Hz}$. However, most bearing fault signals are usually large-parameter signal in which the frequency is larger than $1 \mathrm{~Hz}$, which cannot be processed by the traditional SR. As to this problem, some large-parameter SR (LPSR) methods are proposed such as normalized scale transform SR [4], step-changed SR [5], and re-scaling SR [6]. In essence, these methods transform the large-parameter signal into a proper small-parameter signal or adjust the SR potential parameters to the proper. Normalized scale transform SR will be used in our proposed method.

Most SR methods concern four aspects including SR models, SR potentials, optimization algorithms, and evaluation criterions $[7,8]$. SR models express the SR phenomenon types such as overdamped SR [9], underdamped SR [10], cascaded SR [11], and parallel SR [12]. SR potentials express the SR nonlinear systems such as monostable potential [13], bistable potential [9-12], tristable potential [14], and multistable potential [15]. SR model and SR potential 
focus on the description of SR phenomenon. The underdamped SR with bistable potential will be introduced in our proposed method. Unlike these two aspects, optimization algorithms are used to find the proper parameters adaptively that transform the larger signal to the proper small-parameter signal or adjust the potential parameter to the proper. Many optimization algorithms can be used such as differential evolution algorithm [16-18], cuckoo search $[9,13]$, and Levenberg-Marquardt algorithm [12]. Cuckoo search is a good optimization algorithm which has been used in our previous studies $[9,13]$ and will be used in our proposed.

Evaluation criterions are used to evaluate the SR effect. When optimization algorithms are used to find the optimal SR parameters adaptively, evaluation criterions are necessary. The most commonly used evaluation indexes are signal-to-noise ratio (SNR) and its improved indexes [9-13]. Larger the SNR or its improved indexes are, better the SR effect is. When calculating these indexes, the fault characteristic frequency (FCF) is necessary. However, FCF is not always known because the bearing structure parameters or the rotational speed are not always known. Correlation coefficient (CC) [19] and weighted power spectrum kurtosis (WPSK) [20] are independent of the FCF and avoid the limitation of FCF. Larger the CC or WPSK is, better the SR effect is. However, the two indexes are not always valid to evaluate the SR effect especially when the input SNR is low. Proper synthetic index is made up of multiple single indexes and is more robust than the single index. For example, the WPSK that is made up of CC and power spectrum kurtosis (PSK) is more robust than CC in most cases. In addition, PSK and CC are related to the SNR [20]. Larger the SNR is, the larger the PSK or the CC is. Therefore, the single indexes that constitute the synthetic index should be independent of the FCF and related to the SNR. In this paper, we collect four indicators that are independent on FCF, research their relationship with the SNR, and propose a novel synthetic quantitative index (SQI) instead of SNR.

The remaining sections are arranged as follows. Section 2 will introduce the bearing fault diagnosis framework based on adaptive underdamped SR, underdamped SR, and cuckoo search. Section 3 will introduce the four evaluation indexes that are independent on FCF, analyze their relationship with the SNR, and propose the SQI. Section 4 will verify the novel SQI by numeric simulation and apply the proposed SQI-AUSR to bearing fault diagnosis. Section 5 will summarize this paper.

\section{Bearing Fault Diagnosis Based on Adaptive Underdamped Stochastic Resonance}

2.1. Bearing Fault Diagnosis Framework. A bearing fault diagnosis framework with eight steps is established, as shown in Figure 1.

Step 1: acquire the vibration signal of the bearings. The closer the sensor is to the bearing, the easier the fault is to be detected from the acquired vibration signal.
Step 2: preprocess the signal. Resonance demodulation is applied to preprocess the bearing signal. Then, the Hilbert transform is used to obtain the envelope signal.

Step 3: initialize the parameters. Some parameters of USR and CS should be set.

Step 4: operate USR. The envelope signal is inputted to the USR and the SR output is obtained. And, the USR will be introduced in Section 2.2.

Step 5: evaluate the SR output by an index. The most used index is the SNR. However, the FCF should be known at first. A novel synthetic index that does not need the FCF is proposed in this paper. The novel synthetic index will be introduced in Section 3.

Step 6: execute the termination judgment. If the index reaches the optimum or the iteration reaches the max, the SR output and the optimum parameter will be recorded, and step 8 will be jumped to. Otherwise, step 7 will be carried out.

Step 7: search the optimum parameters by CS. CS is an effective heuristic optimization algorithm, which can help find the optimum USR parameters quickly. It will be introduced in Section 2.3.

Step 8: analyse and diagnose the output. The final SR out will be analyzed and the bearing condition will be diagnosed.

2.2. Underdamped Stochastic Resonance. SR describes an interesting noise-benefit physical phenomenon that the weak signal can be enhanced when the weak signal and proper noise are inputted to a proper nonlinear system. When the damping factor is considered, the underdamped SR (USR) phenomenon can be described as follows:

$$
\frac{\mathrm{d}^{2} x}{\mathrm{~d} t^{2}}+\gamma \frac{\mathrm{d} x}{\mathrm{~d} t}=-\frac{\mathrm{d} U(x)}{\mathrm{d} x}+S_{d}(t)+N(t),
$$

where $x$ is the trajectory of the particle. $\gamma>0$ is the damping factor. When $\gamma=0$, the SR is called overdamped SR (OSR). $S_{\mathrm{d}}$ $(t)=A \sin \left(2 \pi f_{\mathrm{d}} t\right)$ is the weak driving signal with amplitude $A$ and frequency $f_{d} . N(t)=(2 D)^{1 / 2} \varepsilon(t)$ is the Gaussian white noise $(\mathrm{GWN})$ with noise intensity $D . \varepsilon(t)$ is the standard GWN. $U(x)$ is the nonlinear system called potential function, and the common nonlinear system is the bistable potential function as follows:

$$
U(x)=-\frac{a}{2} x^{2}+\frac{b}{4} x^{4}
$$

where both $a>0$ and $b>0$ are the potential parameters of the bistable potential function $U(x)$ whose shape is shown in Figure 2. The bistable potential function $U(x)$ has two minimums located at $\pm x_{m}= \pm(a / b)^{1 / 2}$ and a potential barrier located at $x_{b}=0$ with the height $\Delta U=a^{2} /(4 b)$.

Substituting (2) into equation (1), the bistable USR is obtained as follows:

$$
\frac{\mathrm{d}^{2} x}{\mathrm{~d} t^{2}}=-\gamma \frac{\mathrm{d} x}{\mathrm{~d} t}+a x-b x^{3}+S_{d}(t)+N(t)
$$




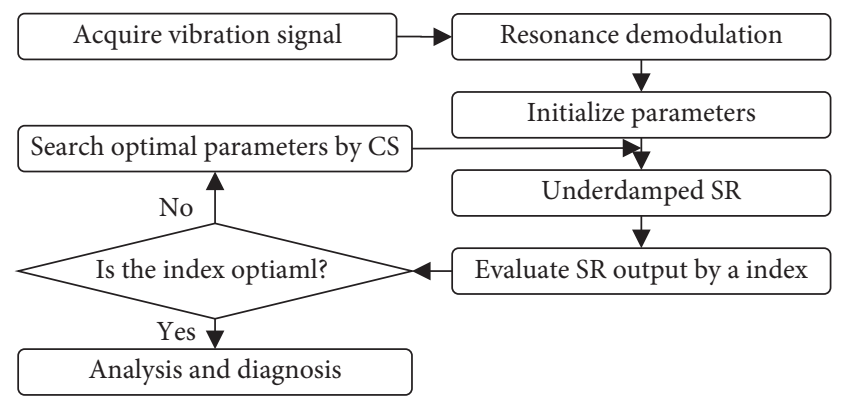

Figure 1: Bearing fault diagnosis framework.

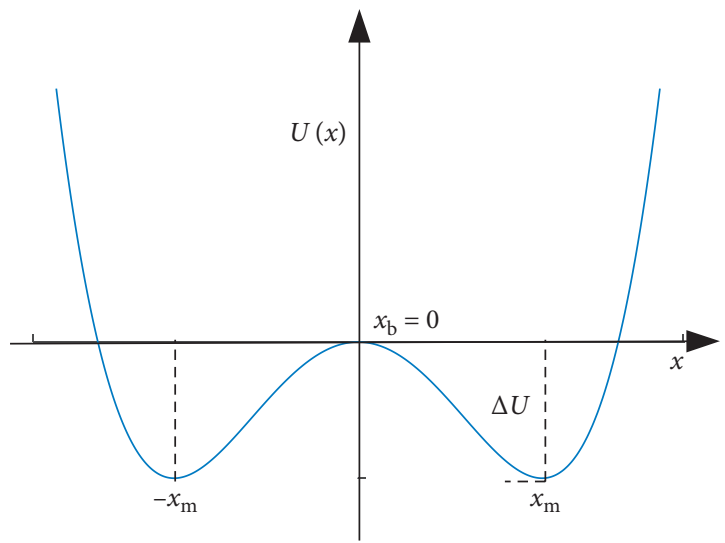

FIGURE 2: The shape of the bistable potential function $(U)(x)$.

Let $z=(a / b)^{1 / 2} x, \tau=a t$, and $S(t)=S_{d}(t)+N(t)$; then, (3) is rewritten as follows:

$$
\frac{\mathrm{d}^{2} z}{\mathrm{~d} \tau^{2}}=\frac{-\gamma(\mathrm{d} z / \mathrm{d} \tau)+z-z^{3}+K S\left(\tau /\left(f_{s} H\right)\right)}{f_{s} H},
$$

where $K=\left(b / a^{3}\right)^{1 / 2}$ is the amplitude gain, $H=a \times h=a / f_{s}$ is the time resolution after transformation, and $f_{s}$ is the sampling frequency. Because the coefficient of $z$ becomes 1 after transformation, this transformation is called normalized scale transform.

After transformation, the current time resolution $H$ is $a$ times of the original, which says that the driving frequency $f_{d}$ is reduced by $a$ times. In addition, the signal amplitude also becomes $K$ times of the original. Therefore, signal $S$ is transformed from a large-parameter signal into a smallparameter signal after transformation, and the LPSR can be realized.

Let $z_{1}=z$ and $z_{2}=\mathrm{d} z_{1} / \mathrm{d} \tau$; equation (4) can be rewritten as follows:

$$
\left\{\begin{array}{l}
\frac{\mathrm{d} z_{1}}{\mathrm{~d} \tau}=z_{2}, \\
\frac{\mathrm{d} z_{2}}{\mathrm{~d} \tau}=\frac{-\gamma z_{2}+z_{1}-z_{1}^{3}+K S\left(\tau / f_{s} H\right)}{f_{s} H} .
\end{array}\right.
$$

The collected vibration signals are all discrete signals. When $S$ is the discrete signal, equation (5) can be solved by equation (6) as follows:

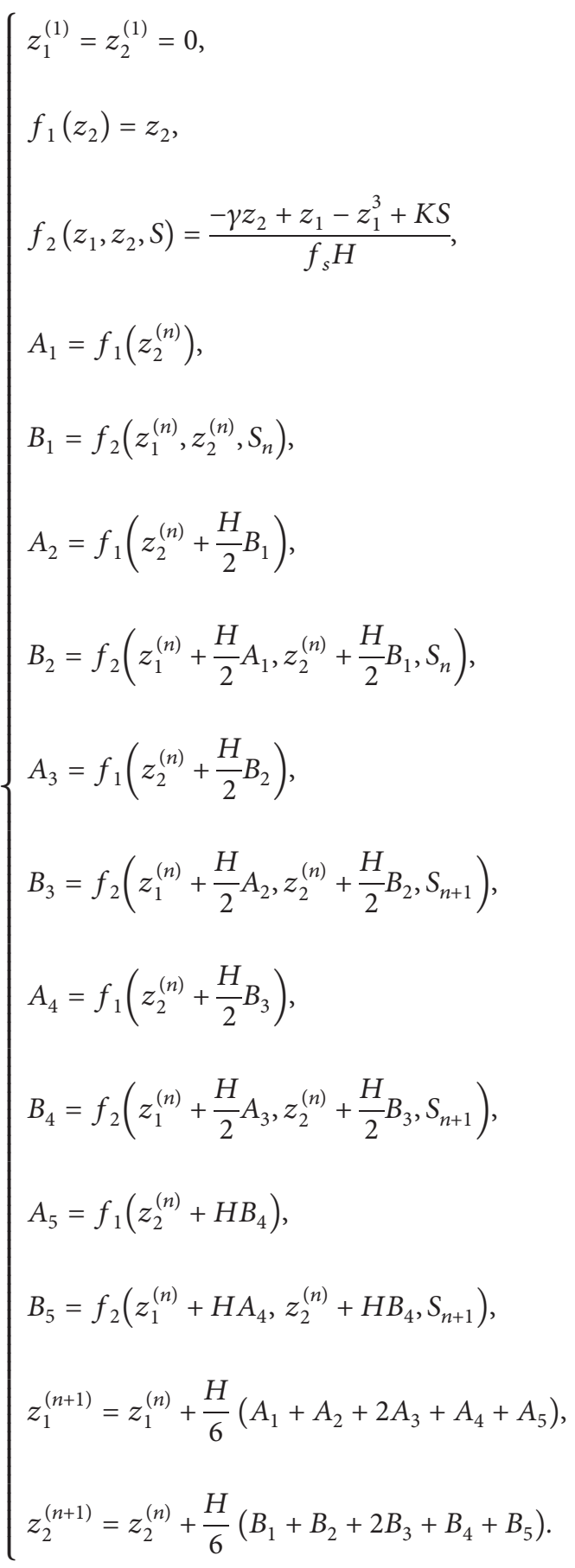

Input signal $S(t)$ is the actual vibration signal and cannot be changed. SR output $z$ is depended on parameters $(H, K, \gamma)$, which can be seen from equation (6). Thus, the adaptive USR focuses on the search of the optimum parameters $(H, K, \gamma)$.

2.3. Cuckoo Search. Cuckoo search (CS) is an effective intelligent optimization algorithm that is designed according to the parasitic breeding strategy of the cuckoos $[9,21]$. This algorithm regards the solutions as the cuckoo eggs, the optimum solution that owns the optimum fitness as the optimum cuckoo egg, and the feasible region of the solutions 
as the cuckoos' search zone. Three ideal assumptions are set up:

The cuckoo lays only one egg at a time and then randomly throws it into the nest.

The optimum eggs at present should be left to the next generation.

The number of nests is immutable. Some cuckoo eggs are found by the host birds with a probability $\mathrm{Pa}=(0$, $1]$, and then, they will be replaced with new eggs.

New solutions are produced from old solutions. The update by Lévy flights is performed as follows[21]:

$$
\left\{\begin{array}{l}
x_{i}^{(t+1)}=x_{i}^{(t)}+s, \\
s \sim R \frac{u}{|v|}\left(x_{i}^{(t)}-x_{\text {best }}^{(t)}\right), \quad i=1,2, \ldots, n \\
u \sim N\left(0, \sigma_{u}^{2}\right), \\
\sigma_{u}^{2}=\left[\frac{\Gamma(1+\beta) \sin ((\pi \beta) / 2)}{\Gamma[(1+\beta) / 2] \beta 2^{(1+\beta) / 2}}\right]^{(1 / \beta)}, \\
v \sim N(0,1), \\
\sigma_{v}^{2}=1
\end{array}\right.
$$

where $x_{i}^{(t+1)}$ is the $i$ th cuckoo egg of the $(t+1)$ th generation, $x_{i}^{(t)}$ is the $i$ th cuckoo egg of the $t$ th generation, $s$ is the step length, $R$ is the step-size scale, $x_{\text {best }}^{(t)}$ is the optimum cuckoo egg among the whole eggs of the $t$ th generation, $u$ and $v$ obey Gaussian distribution, $\Gamma(\cdot)$ is the standard gamma function, $n$ is the number of the nests, and $\beta$ is a constant and equal to 1.5 in this paper.

Based on these three rules and the necessary steps, CS can be summarized as the pseudocode shown in Figure 3.

\section{Novel Synthetic Quantitative Index}

Many indexes can be used to evaluate the SR output. SNR is the most used index. However, the FCF should be known before the calculation of SNR. A novel synthetic index that does not need the known FCF is proposed in this section.

3.1. Traditional Evaluation Indexes. Five traditional evaluation indexes including SNR, power spectrum kurtosis (PSK), kurtosis (K), margin index (MI), and correlation coefficient (CC) are shown in Table 1.

Supposing that the input signal is $x(k)$, the output signal is $y(k)$, the frequency spectrum of $y(k)$ is $Y(k)$, and the power spectrum of $y(k)$ is $P Y(k)$, the spectrums and the five indexes are defined as follows:

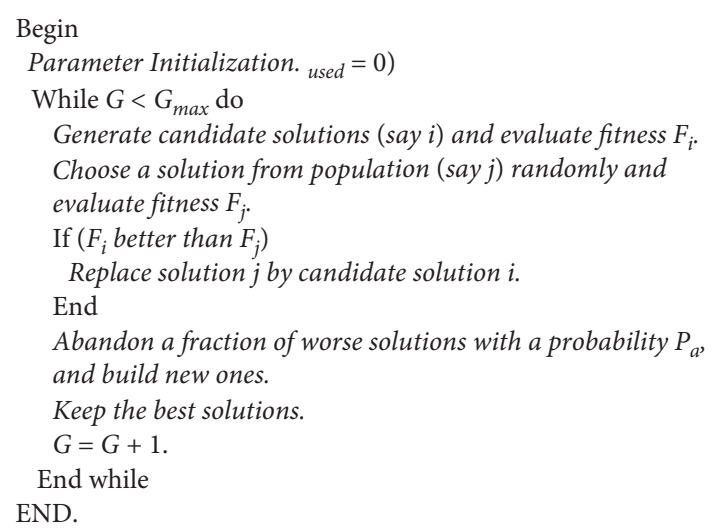

FIgURE 3: Pseudocode of CS.

$$
\begin{aligned}
Y(k) & =\frac{1}{N}\left|\sum_{n=0}^{N-1} y(n) \exp \left(-j \frac{2 \pi k n}{N}\right)\right|, \\
P Y(k) & =\frac{1}{N}\left|\sum_{n=0}^{N-1} y(n) \exp \left(-j \frac{2 \pi k n}{N}\right)\right|^{2}, \\
\mathrm{SNR} & =10 \log \frac{P Y_{d}}{\sum_{i=1}^{(N / 2)} P Y_{i}-P Y_{d}}, \\
\mathrm{PSK} & =\frac{(2 / N) \sum_{i=1}^{(N / 2)}(P Y(i)-\overline{P Y})^{4}}{\left((2 / N) \sum_{i=1}^{(N / 2)}(P Y(i)-\overline{P Y})^{2}\right)^{2}}, \\
K & =\frac{N \sum_{i=1}^{N}\left(y_{i}-\bar{y}\right)^{4}}{\left(\sum_{i=1}^{N}\left(y_{i}-\bar{y}\right)^{2}\right)^{2}}, \\
\mathrm{MI} & =\frac{\max \left|y_{i}\right|}{\left((1 / N) \sum_{i=1}^{N} \sqrt{\left|y_{i}\right|}\right)^{2}}, \\
\mathrm{CC} & =\frac{\sum_{i=1}^{N}\left(x_{i}-\bar{x}\right)\left(y_{i}-\bar{y}\right)}{\sqrt{\sum_{i=1}^{N}\left(x_{i}-\bar{x}\right)^{2} \sum_{i=1}^{N}\left(y_{i}-\bar{y}\right)^{2}}} .
\end{aligned}
$$

3.2. Correlation Analysis by Numeric Simulation. To find the index instead of SNR, the correlations between SNR and the other four indexes are analyzed. Correlation coefficient is a common index that reflects the relationship between two variables. The correlation coefficient of variable $A$ and variable $B$ is defined as follows:

$$
\mathrm{CC}=\frac{\sum_{i=1}^{N}\left(A_{i}-\bar{A}\right)\left(B_{i}-\bar{B}\right)}{\sqrt{\sum_{i=1}^{N}\left(A_{i}-\bar{A}\right)^{2} \sum_{i=1}^{N}\left(B_{i}-\bar{B}\right)^{2}}} .
$$

The closer the $|C C|$ is to 1 , the greater the correlation between $A$ and $B$ is. When CC is smaller than $0, A$ and $B$ are negatively correlated. When $C C$ is larger than 0 , they are positively correlated. 
TABle 1: Tradition signal evaluation indexes.

\begin{tabular}{lc}
\hline Need FCF & No need FCF \\
\hline SNR & PSK, K, MI, CC \\
\hline
\end{tabular}

To analyze the correlations between SNR and the other four indexes, a numeric simulation will be carried out. In the simulation, the driving signal $S_{d}$ is the sine signal with driving frequency $f_{d}=120 \mathrm{~Hz}$ and amplitude $A=1$. The noise is the GWN with the noise intensity from 0.199 to 49.882 (an equal ratio sequence with length 49 and ratio 1.122). Sampling frequency $f_{\mathrm{s}}$ is 8000 . Signal length $N$ is 2048. To reduce the influence of noise randomness, 10 groups of noise with the same intensity were generated. Thus, 490 groups of noise were generated. The mixtures of the driving signal and every noise are regarded as the USR inputs and their SNRs are calculated. These USR inputs are processed by USR, and CS is used to search the optimum parameters $(H, K, \gamma)$, which can maximize the SNR of the USR outputs. The max SNRs of the USR outputs are recorded. Then, PSK, K, and MI of the USR outputs and the CC between input and every USR output are calculated. The CCs are calculated as shown in Table 2. From Table 2, we can find that there is a positive correlation between CC and input SNR, a positive correlation between PSK and output SNR, a negative correlation between $\mathrm{K}$ and output SNR, and a negative correlation between MI and output SNR. And, the four relationships are shown in Figure 4, from which the same conclusion as Table 2 can be drawn. Figure 5 shows the box plots of PSK, K, and MI. From Figure 5, we can find that most of the three indexes are in their own specific small areas.

3.3. Novel Synthetic Quantitative Index. CC, PSK, K, and MI cannot replace SNR singly. Under the comprehensive consideration of these four indexes, a novel synthetical quantitative index (SQI) is constructed as

$$
\mathrm{SQI}=|\mathrm{CC}|\left(\omega_{\mathrm{PSK}} \frac{\mathrm{PSK}}{M_{\mathrm{PSK}}}+\omega_{K} \frac{M_{K}}{K}+\omega_{\mathrm{MI}} \frac{M_{\mathrm{MI}}}{\mathrm{MI}}\right),
$$

where $C C$ is the correlation coefficient between USR input and output. PSK, $\mathrm{K}$, and MI are the power spectrum kurtosis, kurtosis, and margin index of the USR output, respectively. $\omega_{\mathrm{PSK}}=0.862, \omega_{\mathrm{K}}=0.772$, and $\omega_{\mathrm{MI}}=0.830$ are the weights of PSK, K, and MI, respectively, which can be found in Table 2. $M_{\mathrm{PSK}}=1002.227, M_{\mathrm{K}}=1.989$, and $M_{\mathrm{MI}}=2.643$ are the medians of PSK, K, and MI, which can be found in Figure 5.

The larger the SQI is, the better the USR output is. Thus, an objective function for searching the optimum USR parameters can be built as

$$
(H, K, \gamma)=\max \operatorname{SQI}(z)
$$

\section{Verification}

4.1. Verification by Numeric Simulation. The effectiveness of SQI will be analyzed by numeric simulation. SQI will be used as the evaluation index of USR output. For comparison, CC [19] and WPSK [20] are also used as the evaluation index.

In this simulation, the driving signal $S_{\mathrm{d}}$ is the sine signal with driving frequency $f_{d}=120 \mathrm{~Hz}$ and amplitude $A=1$. The noise is the GWN with the noise intensity from 0.250 to 49.882 (an equal ratio sequence with length 24 and ratio 1.250). Sampling frequency $f_{\mathrm{s}}$ is 8000 . Signal length $N$ is 2048. To reduce the influence of noise randomness, 5 groups of noise with the same intensity were generated. Thus, 120 groups of noise were generated. The mixtures of the driving signal and every noise are regarded as the USR inputs. The parameters of CS are set as $H \in[10,200], K \in\left[10^{-10}, 10^{3}\right]$, and $\gamma \in\left[10^{-3}, 2\right]$. The max CC, WPSK, and SQI are searched by $\mathrm{CS}$, respectively, and the SNRs of the corresponding USR outputs are also calculated.

We suppose that the index is successful when the SNR of the corresponding USR output is larger than $-1 \mathrm{~dB}$. The success number and success rate are shown in Table 3. From Table 3, we can find that the success rate based on SQI is up to $94.17 \%$ which is the largest of the three methods. Thus, SQI-AUSR is the best and SQI can replace SNR when FCF is unknown.

The input SNRs versus WPSK, SQI, and their USR output SNRs are shown in Figure 6. With the increase of the input SNRs, both the max WPSK and max SQI rise, as shown in Figures 6(a) and 6(b). However, the SNRs of their corresponding USR outputs are around $[-1,15] \mathrm{dB}$, which means that the USR output SNR has the upper limit. Meanwhile, the faults appear when the input signal is small, as shown in Figures 6(c) and 6(d). However, the fault times of SQI-AUSR are smaller than WPSK-AUSR. It means that SQI-AUSR is better than WPSK-AUSR when the input SNR is small. And, when the input SNR is high, both of these methods are right.

4.2. Verification by Bearing Fault Signals. Two bearing planted-fault tests will be conducted through the test rig, as shown in Figure 7. The planted-fault bearing type is ER-12K, whose main dimensions and FCFs/ $f_{\mathrm{R}}$ are shown in Table 4. The planted fault is a deep groove with $0.5 \mathrm{~mm}$ width located in the inner or outer race. The planted-fault bearing is located in bearing 1 . The motor speed $f_{R}$ is $30 \mathrm{round} / \mathrm{s}$.

The relevant parameters are set as follows: sampling frequency $f_{s}=12.8 \mathrm{kHz}$, signal length $N=8192, H \in\left[10^{-3}\right.$, $\left.10^{2}\right], K \in\left[10^{-3}, 10^{2}\right], \gamma \in\left[10^{-3}, 2\right]$, max generation $G_{\max }=50$, and population size $n=30$. SNR-AOSR [9] and the proposed SQI-AUSR are both used to analyze the fault signals for comparison.

4.2.1. Verification by Outer Race Fault Signal. The outer race fault signal is analyzed at first. $f_{\mathrm{BPFO}}$ is equal to $91.44 \mathrm{~Hz} .30$ order Butterworth band-pass filter with the pass-band [1500, 5000] $\mathrm{Hz}$ is applying to filtering the fault signal. Then, Hilbert transform is used to envelop the filtered signal. The envelope signal is obtained, as shown in Figure 8(a). SNRAOSR and SQI-AUSR are used to process the preprocessed signal, respectively. CS is used to search the optimum 
TABLE 2: Correlation coefficients between SNR of the input or output signals and other four indexes.

\begin{tabular}{lcccc}
\hline & PSK & K & MI & CC \\
\hline Input SNR & 0.370 & -0.351 & -0.537 & 0.981 \\
Output SNR & 0.862 & -0.772 & -0.830 & 0.5345 \\
\hline
\end{tabular}

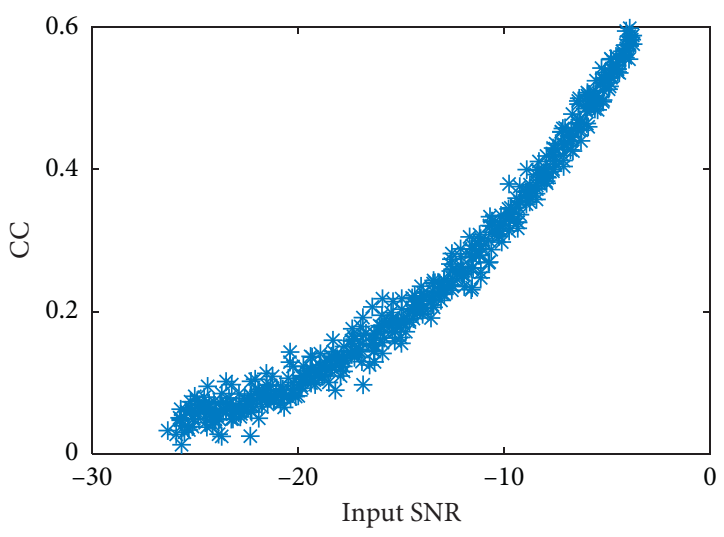

(a)

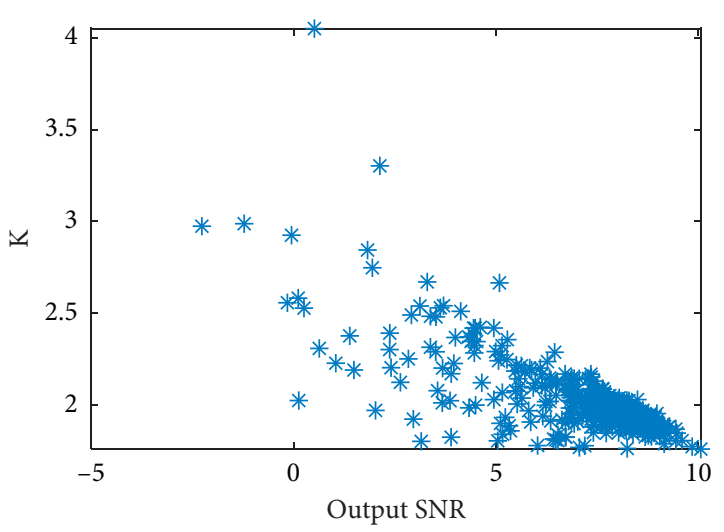

(c)

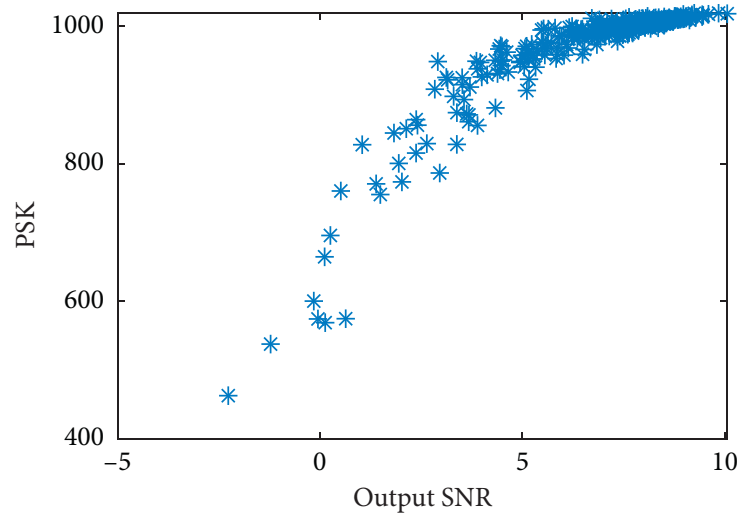

(b)

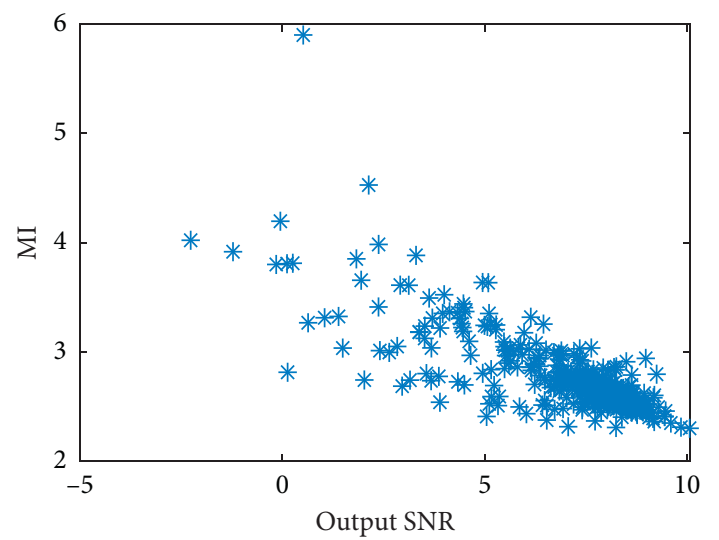

(d)

FIgURE 4: (a) Input SNR versus CC; output SNR versus (b) PSK, (c) K, and (d) MI.

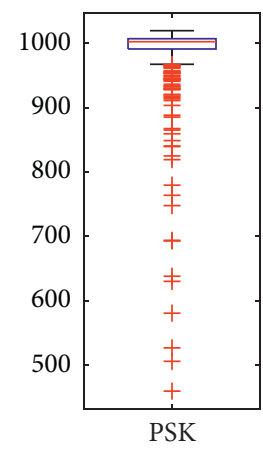

(a)

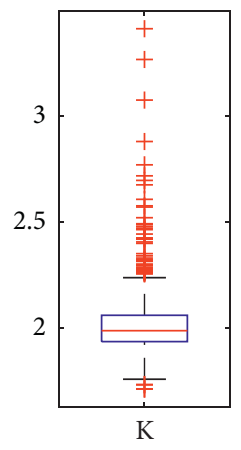

(b)

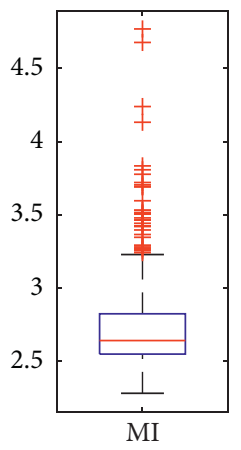

(c)

Figure 5: Box plots: (a) PSK; (b) K; (c) MI. 
TABLE 3: The success times and success rates of CC, WPSK, and SQI.

\begin{tabular}{lccc}
\hline & Success times & Fault times & Success rate $(\%)$ \\
\hline CC-AUSR & 81 & 39 & 67.57 \\
WPSK-AUSR & 107 & 13 & 89.17 \\
SQI-AUSR & 113 & 7 & 94.17 \\
\hline
\end{tabular}

Note. If the SNR of the corresponding USR output is larger than $-1 \mathrm{~dB}$, it is judged as a success.

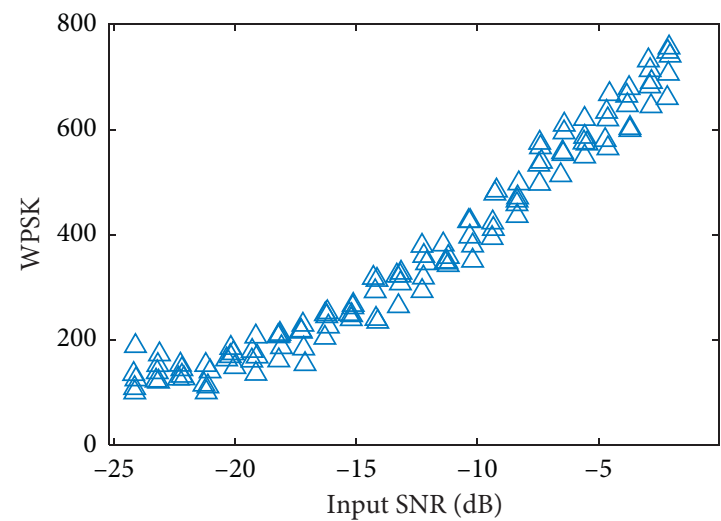

(a)

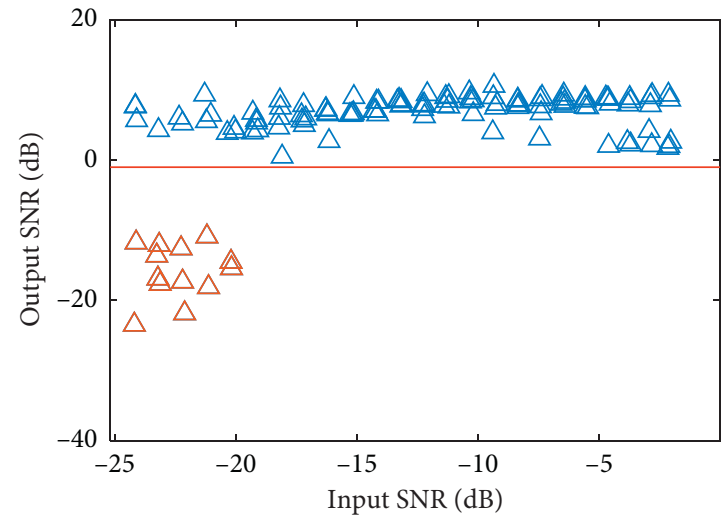

(c)

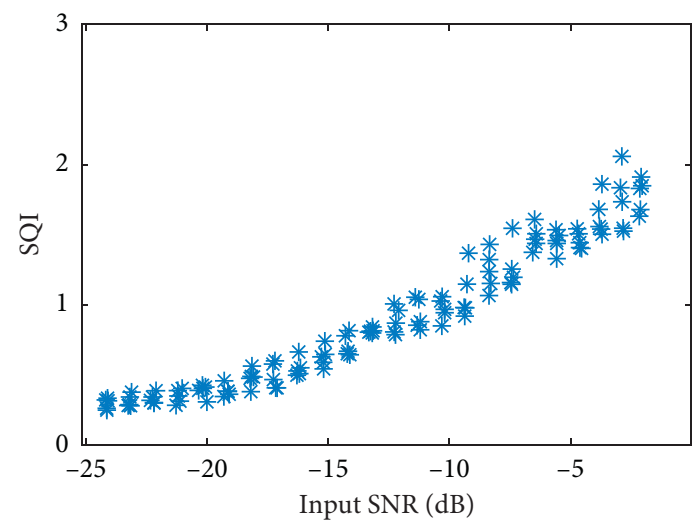

(b)

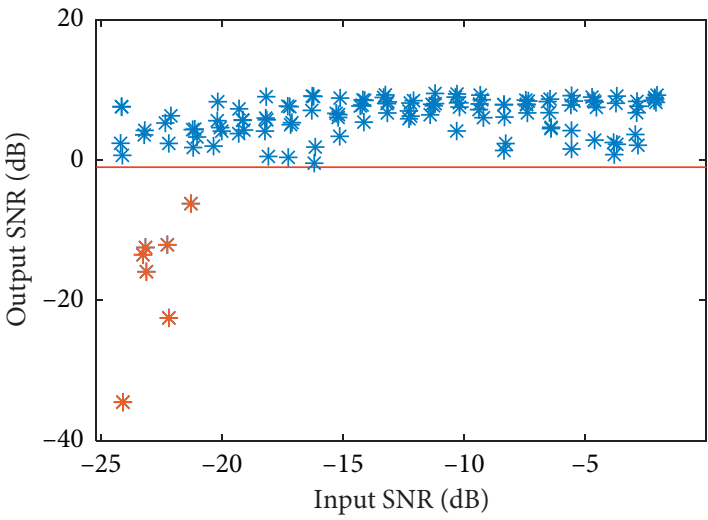

(d)

Figure 6: Input SNRs versus (a) WPSK, (b) SQI, (c) WPSK-AUSR output SNR, and (d) SQI-AUSR output SNR.

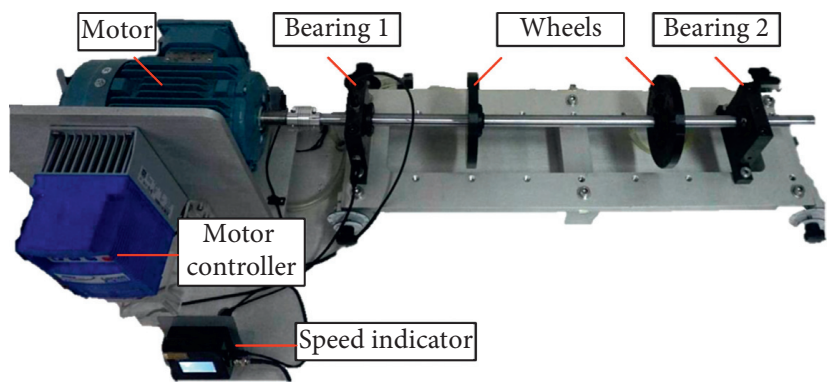

FIgURE 7: Bearing fault test rig.

TABLE 4: The dimensions and characteristic failure parameters of bearing ER-12K.

\begin{tabular}{lcccc}
\hline Ball number & Ball diameter $($ inch) & Pitch diameter (inch & BPFI $\left(f_{\mathrm{R}}\right)$ & BPFO $\left(f_{\mathrm{R}}\right)$ \\
\hline 8 & 0.3125 & 1.318 & 4.950 & 3.048 \\
\hline
\end{tabular}

Note. BPFI means the ball pass frequency inner race; BPFO means the ball pass frequency outer race; $f_{\mathrm{R}}$ is the bearing rotation speed. 

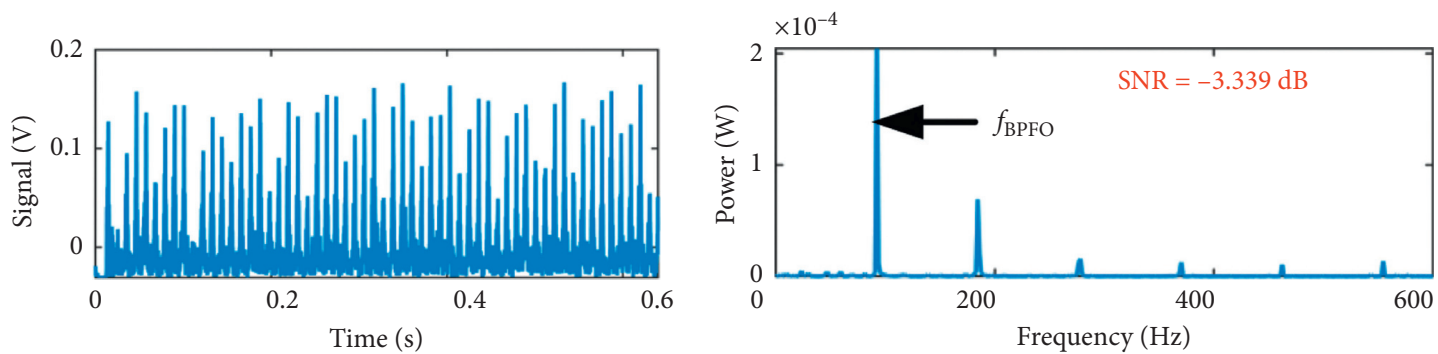

(a)
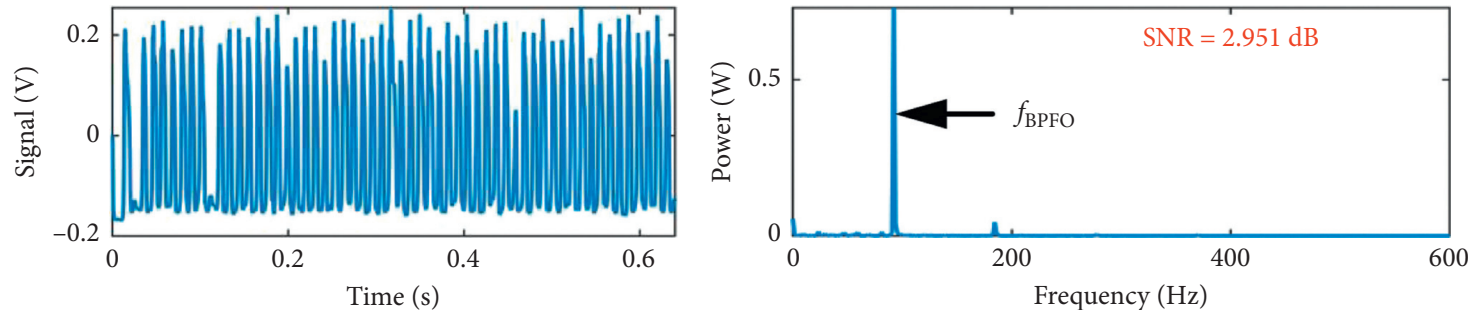

(b)
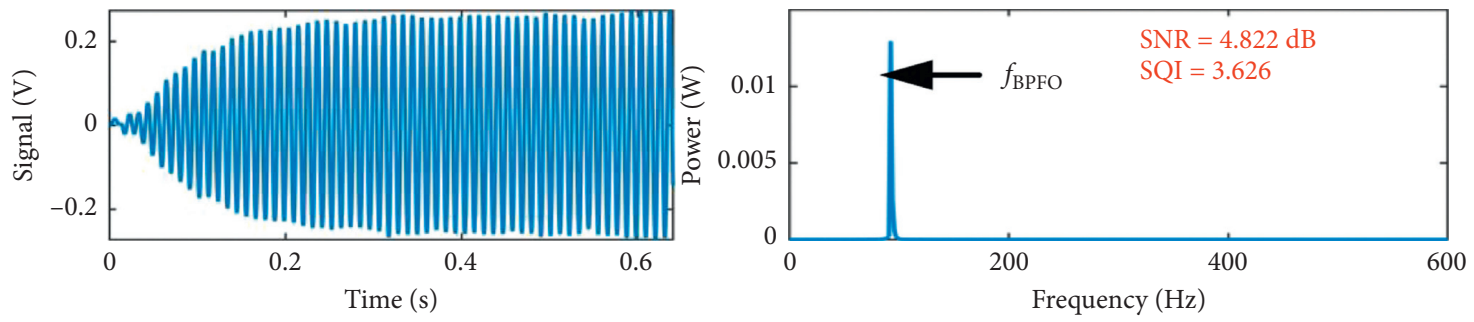

(c)

Figure 8: Analyzed results of the outer race fault signal: (a) envelope signal; (b) SNR-AOSR output with $(H),(K)=(0.0898,100)$; (c) SQIAUSR output with $((H),(K), \gamma)=(28.657,0.287,2)$.

TABLE 5: The SNRs of the preprocessed signal, SNR-AOSR output, and SQI-AUSR output.

\begin{tabular}{lccr}
\hline Fault location & & SNR (dB) & \\
& Preprocessed signal & SNR-AOSR output & SQI-AUSR output \\
\hline Outer race & -3.339 & -2.951 & 4.822 \\
Inner race & -10.300 & -7.138 & 0.946 \\
\hline
\end{tabular}

Note. The preprocessed signal is the SR input. For example, the preprocessed signal is the envelope signal for the outer race fault case.

parameters. Their optimum SR outputs are shown in Figures $8(\mathrm{~b})$ and $8(\mathrm{c})$. The SNRs of the preprocessed signal, SNR-AOSR output, and SQI-AUSR output are shown in Table 5. It is obvious that both the output SNRs are larger than the preprocessed signal, which means that SR can enhance the weak signal. The SQI-AUSR output SNR $(4.822 \mathrm{~dB})$ is higher than the SNR-AOSR output SNR (2.951 dB). Consequently, SQI-AUSR is better than SNR-AOSR, and the proposed SQI is practicable in this case.

4.2.2. Verification by Inner Race Fault Signal. Then, the inner race fault signal is analyzed. $f_{\mathrm{BPFI}}$ is equal to $148.50 \mathrm{~Hz}$. The same way is used to preprocess the fault signal. And, the obtained envelope signal is shown in Figure 9(a). Because the harmonics of the rotation frequency $f_{\mathrm{R}}$ (marked by the dotted box in Figure $9(\mathrm{a})$ ) are intense which can is adverse for SR effect, they removed by the spectrum editing technology, as shown in Figure 9(b) [9]. SNR-AOSR and SQI-AUSR are used to process the preprocessed signal, respectively. CS is used to search the optimum parameters. Their optimum SR outputs are shown in Figures 9(c) and 9(d). The SNRs of the preprocessed signal, SNR-AOSR output, and SQI-AUSR output are shown in Table 5. It is obvious that both the output SNRs are larger than the preprocessed signal, which means that SR can enhance the weak signal. The SQI-AUSR output SNR $(0.946 \mathrm{~dB})$ is much higher than the SNR-AOSR output SNR (-7.138 dB). Consequently, SQIAUSR is better than SNR-AOSR and the proposed SQI is practicable in this case. In Figure 9(c), the low-frequency components that are lower than $f_{\mathrm{BPFI}}$ are also enhanced, which means that SNR-AOSR is like a low-pass filter that cannot filter the low-frequency components. However, SQI-AUSR filters not only the high-frequency 

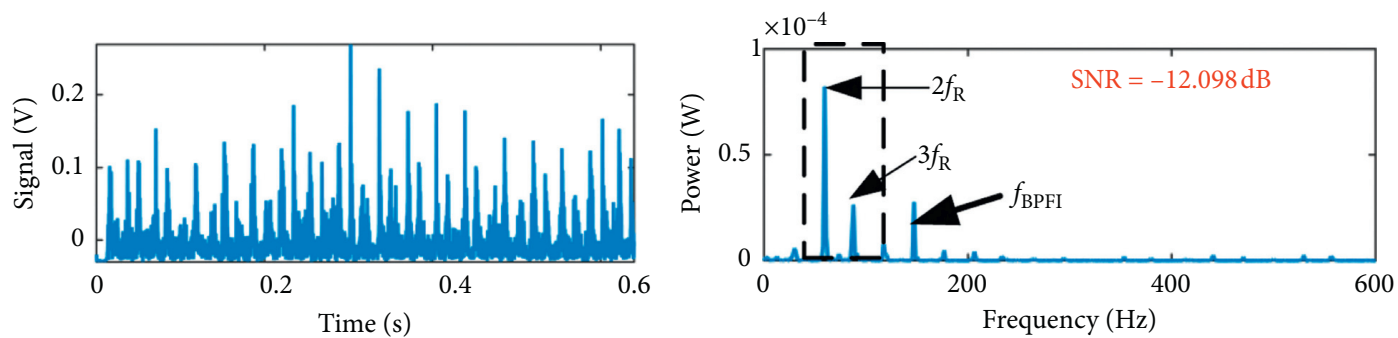

(a)
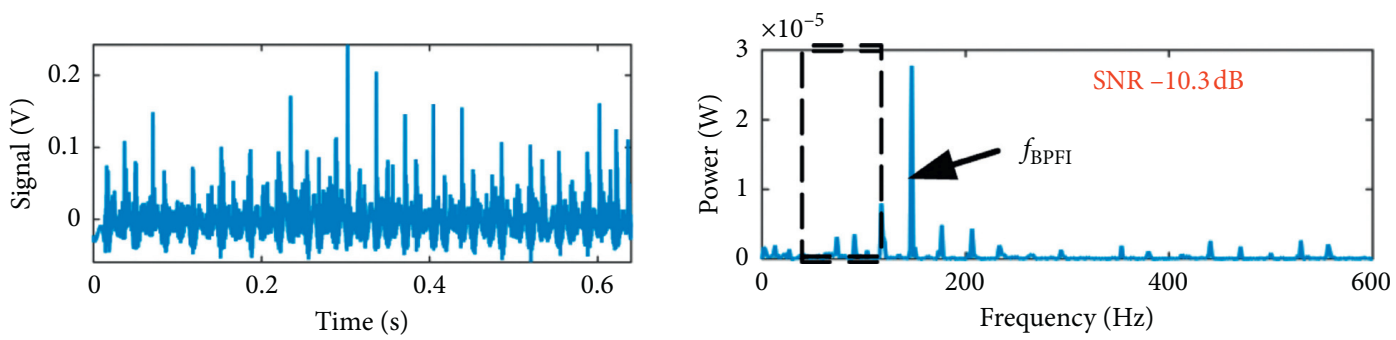

(b)
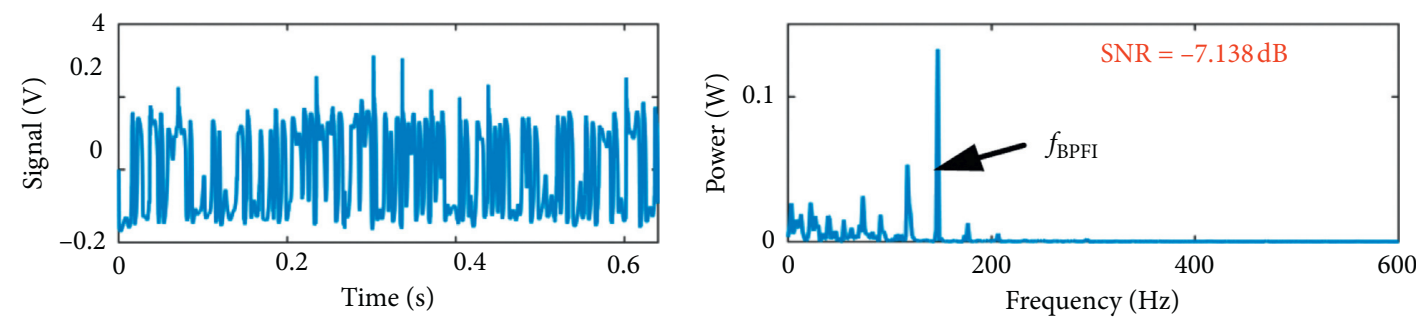

(c)
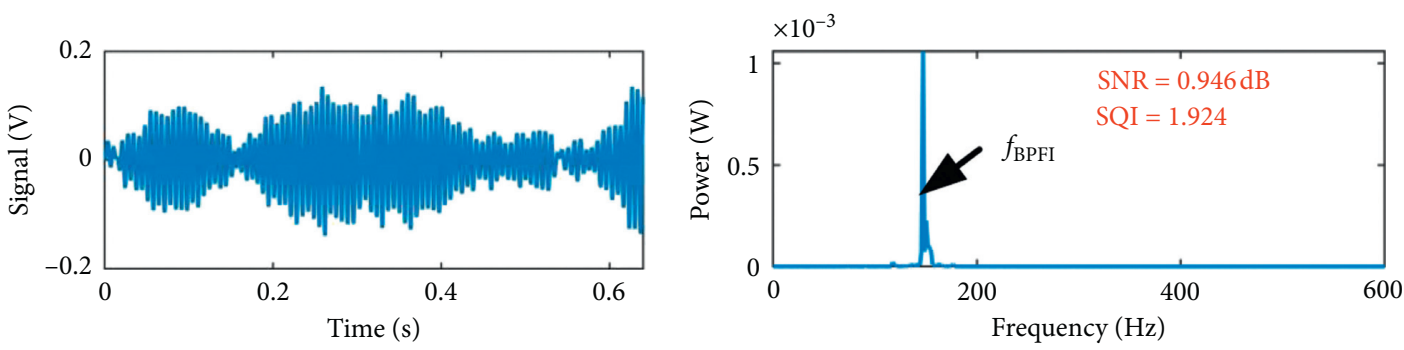

(d)

FIGURE 9: Analyzed results of the inner race fault signal: (a) envelope signal; (b) envelope signal after removing the rotation frequency $f_{\mathrm{R}}$ and its harmonic $2 f_{\mathrm{R}} ;(\mathrm{c})$ SNR-AOSR output with $[(H),(K)]=[0.140,100]$; (d) SQI-AUSR output with $[(H),(K), \gamma]=[72.629,0.594,1.996]$.

components but also the low-frequency components, which means that it is like a band-pass filter.

\section{Conclusions}

A novel synthetic quantitative index-based adaptive underdamped stochastic resonance (SQI-AUSR) is proposed for bearing fault diagnosis. Numeric simulations and case verifications prove that (1) SQI-AUSR can detect the weak signal even when the FCF is unknown, (2) the proposed SQI has more effective than CC or WPSK for realizing the SR, and (3) SQI-AUSR has better capability than SNR-AOSR for the bearing fault diagnosis.

\section{Data Availability}

The experiment data used to support the findings of the study are obtained from the corresponding author upon request.

\section{Conflicts of Interest}

The authors declare that they have no conflicts of interest.

\section{Acknowledgments}

This work was supported by the Reliability Centered Maintenance Laboratory of Shijiazhuang Branch of Army Engineering University and the National Natural Science Foundation of China under Grant nos. 71871220 and 71871219. 


\section{References}

[1] N. Bessous, S. E. Zouzou, W. Bentrah, S. Sbaa, and M. Sahraoui, "Diagnosis of bearing defects in induction motors using discrete wavelet transform," International Journal of System Assurance Engineering and Management, vol. 9, no. 2, pp. 335-343, 2018.

[2] S. N. Chegini, A. Bagheri, and F. Najafi, "Application of a new EWT-based denoising technique in bearing fault diagnosis," Measurement, vol. 144, pp. 275-297, 2019.

[3] T. Gong, X. Yuan, Y. Yuan, X. Lei, and X. Wang, “Application of tentative variational mode decomposition in fault feature detection of rolling element bearing," Measurement, vol. 135, pp. 481-492, 2019.

[4] X. Zhang, N. Hu, Z. Cheng, and L. Hu, "Enhanced detection of rolling element bearing fault based on stochastic resonance," Chinese Journal of Mechanical Engineering, vol. 25, no. 6, pp. 1287-1297, 2012.

[5] Z. Zhang, D. Wang, T. Wang, J. Lin, and Y. Jiang, "Selfadaptive step-changed stochastic resonance using particle swarm optimization," Journal of Vibration and Shock, vol. 32, no. 19, pp. 125-130, 2013.

[6] J. Liu, Y. Leng, Z. Lai, and S. Fan, "Multi-frequency signal detection based on frequency exchange and re-scaling stochastic resonance and its application to weak fault diagnosis," Sensors, vol. 18, no. 5, p. 1325, 2018.

[7] Z. Qiao, Y. Lei, and N. Li, "Applications of stochastic resonance to machinery fault detection: a review and tutorial," Mechanical Systems and Signal Processing, vol. 122, pp. 502536, 2019.

[8] S. Lu, Q. He, and J. Wang, "A review of stochastic resonance in rotating machine fault detection," Mechanical Systems and Signal Processing, vol. 116, pp. 230-260, 2019.

[9] K. Chi, J. Kang, X. Zhang, and Z. Yang, "bearing fault diagnosis based on stochastic resonance with cuckoo search," International Journal of Performability Engineering, vol. 14, no. 3, pp. 413-424, 2018.

[10] S. Lu, Q. He, and F. Kong, "Effects of underdamped stepvarying second-order stochastic resonance for weak signal detection," Digital Signal Processing, vol. 36, pp. 93-103, 2015.

[11] J. Li, Y. Zhang, and P. Xie, "A new adaptive cascaded stochastic resonance method for impact features extraction in gear fault diagnosis," Measurement, vol. 91, pp. 499-508, 2016.

[12] L. Xiao, X. Zhang, S. Lu, T. Xia, and L. Xi, "A novel weak-fault detection technique for rolling element bearing based on vibrational resonance," Journal of Sound and Vibration, vol. 438, pp. 490-505, 2019.

[13] J. Kang, K. Chi, X. Die, Z. Wu, and X. Su, "Application of adaptive mono-stable stochastic resonance based on cuckoo search for incipient fault diagnosis of rolling element bearings," in Proceedings of the 2018 Prognostics and System Health Management Conference, pp. 468-473, Chongqing, China, 2018.

[14] S. Lu, Q. He, H. Zhang, S. Zhang, and F. Kong, "Note: signal amplification and filtering with a tristable stochastic resonance cantilever," Review of Scientific Instruments, vol. 84, no. 2, 2013.

[15] P. Shi, S. An, P. Li, and D. Han, "Signal feature extraction based on cascaded multi-stable stochastic resonance denoising and EMD method," Measurement, vol. 90, pp. 318-328, 2016.

[16] W. Deng, J. Xu, X.-Z. Gao, and H. Zhao, "An enhanced MSIQDE algorithm with novel multiple strategies for global optimization problems," IEEE Transactions on Systems, Man, and Cybernetics: Systems, pp. 1-10, 2020.

[17] W. Deng, J. Xu, Y. Song, and H. Zhao, "Differential evolution algorithm with wavelet basis function and optimal mutation strategy for complex optimization problem," Applied Soft Computing, vol. 100, Article ID 106724, 2020.

[18] W. Deng, H. Liu, J. Xu, H. Zhao, and Y. Song, "An improved quantum-inspired differential evolution algorithm for deep belief network," IEEE Transactions on Instrumentation and Measurement, vol. 69, no. 10, pp. 7319-7327, 2020.

[19] P. Krauss, C. Metzner, A. Schilling et al., "Adaptive stochastic resonance for unknown and variable input signals," Scientific Reports, vol. 7, no. 1, pp. 1-8, 2017.

[20] J. Wang, Q. He, and F. Kong, "Adaptive multiscale noise tuning stochastic resonance for health diagnosis of rolling element bearings," IEEE Transactions on Instrumentation and Measurement, vol. 64, no. 2, pp. 564-577, 2015.

[21] X.-S. Yang and S. Deb, "Multiobjective cuckoo search for design optimization," Computers \& Operations Research, vol. 40, no. 6, pp. 1616-1624, 2013. 